\title{
Impacto da cárie dentária na qualidade de vida de pré-escolares mensurado pelo questionário PedsQL
}

\author{
Impact of dental caries on the quality of life of preschoolers \\ measured by PedsQL questionnaire
}

\author{
Adriana Vasconcelos da Nóbrega (http://orcid.org/0000-0002-6781-6042) ${ }^{1}$ \\ Lucia de Fátima Almeida de Deus Moura (http://orcid.org/0000-0002-4112-1533) ${ }^{2}$ \\ Natália Silva Andrade (http://orcid.org/0000-0001-5945-8401) ${ }^{1}$ \\ Cacilda Castelo Branco Lima (http://orcid.org/0000-0002-2977-6035) ${ }^{1}$ \\ Danielle Gomes Dourado (http://orcid.org/0000-0002-0730-618X) ${ }^{3}$ \\ Marina de Deus Moura de Lima (http://orcid.org/0000-0002-7641-6331) ${ }^{4}$
}

${ }^{1}$ Universidade Federal do

Piauí (UFPI). Campus

Universitário Ministro

Petrônio Portella,

Ininga. 64049-550

Teresina PI Brasil.

nobregaav@gmail.com

${ }^{2}$ Departamento de Patologia

e Clínica Odontológica,

Universidade Federal do

Piauí (UFPI). Teresina PI

Brasil.

${ }^{3}$ Centro de Ciências da

Saúde, Universidade Federal

do Piauí (UFPI). Teresina

PI Brasil.

${ }^{4}$ Departamento de Patologia

e Clínica Odontológica,

Universidade Federal do

Piauí (UFPI). Teresina PI

Brasil.

\begin{abstract}
The objective of this study was to evaluate the impact of dental caries on the quality of life of preschool children. The population was made up of preschoolers aged 5 years, enrolled in public and private institutions of Teresina, PI, Brazil. The Pediatric Quality of Life Inventory (Peds $\left.Q L^{\mathrm{TM}}\right)$ was applied to children and caregivers, as well as a socioeconomic-demographic questionnaire for those responsible. In the dental examination the ceod index was used to evaluate the caries experience. Of the 566 children examined, $50.2 \%$ presented with caries experience. It was observed an association between caries experience and poorer quality of life in the oral health domain in the children's perception (RR $=0.981,95 \% C I=0.97-0.99)$ and parents $(R R$ $=0.955,95 \% C I=0,94-0.97)$. According to the children's perception, caries in the posterior teeth was associated with poorer quality of life in the physical capacity domain $(R R=0.985,95 \% C I=$ 0.97-0.99). It was concluded that caries experience had a negative impact on the OHRQoL (oral health-related quality of life) in children's oral health according to the perception of children and parents. Caries located in molars had a negative impact on HRQOL in the physical ability domain according to the children's perception.
\end{abstract}

Keywords Collective health, Epidemiology, Dental caries.
Resumo O objetivo do estudo foi avaliar o impacto da cárie dentária na qualidade de vida de pré-escolares. A população foi constituída por pré -escolares com idade de 5 anos, matriculados em instituições públicas e privadas de Teresina, PI, Brasil. Instrumento de qualidade de vida validado para população brasileira (Pediatric Quality of Life Inventory - Peds $Q L^{\mathrm{TM}}$ ) foi aplicado para as crianças e responsáveis, além de questionário socioeconômico-demográfico para os responsáveis. No exame dentário o indice ceod foi utilizado para avaliar a experiência de cárie. Das 566 crianças examinadas, 50,2\% apresentaram experiência de cárie. Foi observada associação entre experiência de cárie e pior qualidade de vida no dominio de saúde bucal na percepção das crianças $(R R=$ 0,981; IC95\% =0,97-0,99) e dos pais $(R R=0,955$; IC95\% = 0,94-0,97). De acordo com a percepção das crianças, cárie em dentes posteriores foi associada a pior qualidade de vida no domínio capacidade física $(R R=0,985 ; I C 95 \%=0,97-0,99)$. Concluiu-se que experiência de cárie impactou negativamente na QVRSB (qualidade de vida relacionada à saúde bucal) no domínio saúde bucal da criança de acordo com a percepção das crianças e dos pais. Cárie localizada em molares impactou negativamente na QVRSB no domínio capacidade física segundo a percepção das crianças.

Palavras-chave Saúde coletiva, Epidemiologia, Cárie dentária. 


\section{Introdução}

No Brasil, tem sido observado um declínio na prevalência de cárie dentária nas últimas décadas. Esse fenômeno tem sido atribuído, principalmente, à ampliação da disponibilidade e utilização de produtos fluoretados por grande parte das populações e, também, ao enfoque atual de promoção e prevenção em saúde das políticas em saúde bucal ${ }^{1-4}$. Contudo, a cárie ainda é uma doença prevalente e desigualmente distribuída e seu controle representa um desafio para a saúde pública. Fatores socioeconômicos têm sido associados tanto à experiência de cárie quanto à sua distribuição entre crianças ${ }^{5,6}$.

A maioria dos estudos realizados em Odontologia tem enfoque quantitativo, avaliando presença e gravidade das doenças, sem avaliar como esses problemas interferem na qualidade de vida dos indivíduos que as possuem em relação a dor, desconforto e impacto psicológico ${ }^{7,8}$.

$\mathrm{O}$ conceito de qualidade de vida relacionada à saúde bucal (QVRSB) se refere ao impacto que a saúde bucal ou a doença tem sobre o desempenho de atividades diárias do indivíduo, o bem-estar ou qualidade de vida9. A avaliação da QVRSB é uma importante ferramenta de saúde, tanto na avaliação clínica tradicional como em pesquisas $^{10,11}$. Estudos que avaliam o impacto da saúde bucal na qualidade de vida de crianças podem contribuir para o planejamento de políticas públicas de saúde que visam a diminuir as desigualdades sociais e que devem estar pautados no conhecimento das necessidades da população, correlacionando causas.

Uma parcela significativa desses estudos utiliza como metodologia questionários específicos não abrangendo aspectos sistêmicos relacionados às condições gerais de saúde das crianças e cuidadores pesquisados, limitando o processo analítico sobre os domínios gerais de saúde. No estudo proposto, foi aplicado um questionário junto aos responsáveis para obtenção de dados socioeconômico-demográficos. Instrumento de qualidade de vida validado para população brasileira (Pediatric Quality of Life Inventory - PedsQL ${ }^{\mathrm{TM}}$ ) foi aplicado para as crianças e seus responsáveis ${ }^{12}$.

Além disso, é importante explorar as percepções dos pais sobre a saúde bucal de seus filhos, visto que podem afetar nos cuidados preventivos e na utilização de serviços odontológicos que estas crianças recebem ${ }^{13}$. Estudos com essa abordagem ainda são escassos, no público infantil ${ }^{1-4}$. Esse é o primeiro estudo a utilizar o questionário PedsQL ${ }^{\mathrm{TM}}$ em pré-escolares. Desta forma, este es- tudo tem por objetivo avaliar o impacto da cárie dentária na qualidade de vida de pré-escolares de Teresina-PI, Brasil, sendo relevante para a academia e para a sociedade. A hipótese do estudo é que pré-escolares com cárie dentária tem maior impacto negativo na qualidade de vida.

\section{Métodos}

Esta pesquisa foi submetida e aprovada pelo Comitê de Ética em Pesquisa (CEP) da Universidade Federal do Piauí, obedecendo às diretrizes da Resolução 466/12 do Conselho Nacional de Saúde ${ }^{14}$.

\section{Tipo de Estudo}

Trata-se de um estudo transversal. Foram utilizadas informações de um banco de dados do projeto de pesquisa intitulado "Impacto dos defeitos de desenvolvimento do esmalte na qualidade de vida de pré-escolares". O artigo foi redigido de acordo com os guidelines do "Strengthening the reporting of observational studies in epidemiology" (STROBE). A coleta dos dados foi realizada no período de junho a dezembro de 2014.

\section{População e Amostra}

A população do estudo foi constituída por pré-escolares com idade de 5 anos, matriculados em instituições públicas municipais e particulares do município de Teresina-PI, perfazendo um total de 7792 pré-escolares matriculados. A amostra do estudo foi do tipo probabilística e aleatória, sendo calculada utilizando a equação $n=z^{2} \cdot p \cdot(1-p) /$ $e^{2}$, em que " $z$ " é o quantil da distribuição normal (para um intervalo de confiança de $95 \%$, tem-se $z=1,96)$; "p" é a variação estimada para impacto negativo da cárie dentária na qualidade de vida (50\%); "e" é a margem de erro considerada (5\%). Em seguida foi aplicada a correção de Cochran para populações finitas, $n=n 0 /(1+n 0 / N)$, onde, n0 é o tamanho inicial da amostra; N: é o tamanho da população (7792 pré-escolares). Foi obtida uma amostra ideal para o desenvolvimento do estudo de 365 crianças, por se tratar de uma amostra em múltiplos estágios foi corrigido o efeito do desenho (design effect) por um fator de 1,5 (365 x 1,5 $=547$ ). A fim de minimizar as perdas durante o levantamento dos dados, foi aumentado o tamanho da amostra em $10 \%$, sendo obtida uma amostra final de $602(547+55=602)$ pré-escolares.

Foi realizado sorteio das pré-escolas por superintendência regional (centro/norte, sul, leste 
e sudeste) de Teresina, Piauí e por tipo de pré-escola (pública e privada) a partir da lista nominal fornecida pela Secretaria Municipal de Educação e Cultura de Teresina-PI e pelo Sindicato das Escolas Particulares de Teresina-PI. Em cada escola as crianças foram sorteadas a partir da lista de frequência escolar. Na situação de não permissão por parte da pré-escola em participar da pesquisa, outra da mesma superintendência foi sua substituta. Caso a pré-escola sorteada não fosse capaz de completar a amostra definida, outra foi sorteada, até que o número de crianças objetivado fosse atingido. Foram inclusas no estudo 19 pré-escolas públicas municipais e 21 pública.

\section{Critérios de Inclusão e Exclusão}

Foram incluídos pré-escolares com 5 anos de idade que estavam na fase de dentição decídua e que possuíam, no mínimo, dois incisivos decíduos presentes em cada arco dentário.

Foram excluídos pré-escolares em fase de dentição permanente e crianças com necessidades especiais pela impossibilidade que estas têm para responder aos questionários de qualidade de vida que avalia a auto percepção das crianças na faixa etária analisada, uma exigência do instru- mento utilizado. A necessidade especial da criança foi informada pelas professoras.

\section{Coleta e Análise dos Dados}

Os dados foram coletados a partir da aplicação de questionários sociodemográficos aos responsáveis das crianças, do instrumento de qualidade de vida validado (aplicado aos responsáveis e às crianças) e do exame clínico intrabucal (Figura 1).

\section{Variáveis Utilizadas no Estudo}

Variáveis coletadas no questionário respondido pelos pais sobre aspectos sociodemográficos: sexo, convive com pai e mãe na mesma casa, tipo de escola, higienização dos dentes por dia, ingestão de guloseimas por semana, história de trauma dentário anterior. Relacionado aos responsáveis: renda familiar, escolaridade da mãe e escolaridade do pai.

Quanto às variáveis verificadas no exame clínico bucal têm-se: experiência de cárie, localização necessita de tratamento. Variáveis analisadas no instrumento validado: capacidade física, aspecto emocional, aspecto social, atividade escolar, saúde bucal da criança.

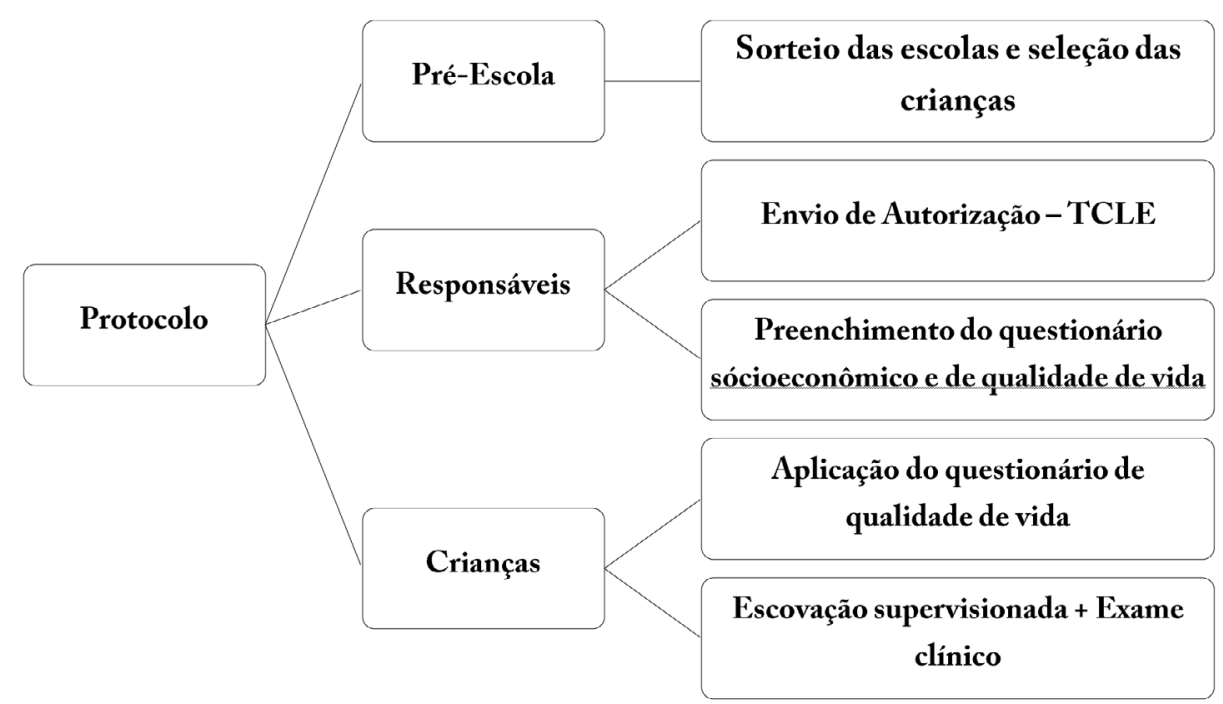

Figura 1. Fluxograma do estudo 


\section{Calibração}

O exercício de calibração foi conduzido em duas fases. As fases teórica e prática envolveram a discussão dos critérios diagnósticos para cárie de acordo com o índice ceod. Nesse estágio, foram analisadas fotos de dentes com e sem cárie de pacientes atendidos na Clínica Infantil da UFPI. A fase teórico-prática da calibração foi coordenada por especialista em odontopediatria, considerada padrão-ouro para a avaliação. Quando o examinador e o padrão-ouro concordavam em $80 \%$ das avaliações, a segunda fase da calibração foi iniciada. A segunda fase envolveu exames clínicos de 60 pacientes com intervalo de 15 dias entre os exames. O kappa intra-examinador observado foi de 0,86 e o inter-examinador foi 0,82 .

\section{Estudo Piloto}

Inicialmente foi realizado um estudo piloto em 3 creches ( 2 públicas e 1 privada), correspondendo a $10 \%$ da amostra final (60 crianças). Estas crianças não foram incluídas na amostra final da pesquisa. Os objetivos do estudo piloto foram a adequação da metodologia da pesquisa e avaliação de confiabilidade e validade do questionário de qualidade de vida para este estudo por meio dos testes alpha de Cronbach e teste-reteste (Coeficiente de Variação Intra-classe) ${ }^{15}$. Os valores observados foram 0.724 para o alpha de Crombach e test-retest $=0.726$. Não foram necessárias alterações no projeto inicial.

\section{Características socio-econômico- demográficas}

Através de um questionário preenchido pelos responsáveis foram coletadas características sociodemográficas da amostra como informações sobre gênero, renda familiar, escolaridade da mãe e do pai, frequência de escovação, tipo de escola e história de traumatismo dentário.

\section{Questionário de Qualidade de Vida (PedsQL)}

A qualidade de vida geral e relacionada à saúde bucal foi avaliada utilizando-se a versão brasileira do questionário "Pediatric Quality of Life Inventory” (PedsQL) aplicado aos responsáveis e às crianças de 5 anos de idade ${ }^{12}$. $\mathrm{O}$ questionário PedsQL $L^{T M}$ Oral Health Scale, PedsQL ${ }^{T M} 4.0$ Generic Core Scales possui duas versões, uma direcionada a avaliar a autopercepção das crianças na faixa etária de 5 a 7 anos e outra para percepção dos responsáveis; ambos ponderam sobre aspectos multidimensionais da saúde da criança.

O questionário PedsQL $L^{T M} 4.0$ Generic Core Scales é composto por 23 itens divididos em quatro domínios: capacidade física (8 itens), aspecto emocional (5 itens), aspecto social (5 itens), e atividade escolar ( 5 itens) e o questionário Peds $Q L^{T M}$ Oral Health Scale é composto por 5 itens. Como respostas no questionário dos responsáveis, foi utilizada uma escala de 5 pontos $(100=$ nunca é um problema; 75 = quase nunca é um problema; 50 = às vezes é um problema; $25=$ muitas vezes é um problema; 0 = quase sempre é um problema). Para facilitar o uso dessa escala para as crianças, foi utilizada escala hedônica facial simplificada, contendo apenas 3 respostas, que corresponderam a: $100=$ nunca é problema, $50=$ às vezes é um problema, 0 = quase sempre é um proble$\mathrm{ma}^{15,16}$. Quanto menor o escore no questionário, pior a qualidade de vida, pontuações maiores são indicativas de melhor qualidade de vida.

\section{Exame clínico bucal}

$\mathrm{Na}$ última etapa de coleta de dados, foi realizado exame clínico para avaliar experiência de cárie dentária. Esta avaliação clínica foi realizada dentro da própria instituição de ensino na qual a criança estava matriculada por um único examinador previamente treinado e calibrado para cárie dentária (valor do Índice Kappa maior que $0,60)$. Inicialmente, foi realizada a higienização dos dentes com escova dental e dentifrício fluoretado. $\mathrm{O}$ exame foi realizado em sala de aula e foi utilizada lâmpada artificial (Pelican ${ }^{\circledR}$ model - Startec with 127V, São Paulo, Brazil). As crianças sentavam na cadeira e suas cabeças eram posicionadas no colo do examinador. Foi utilizada gaze estéril para secar os dentes e o exame clínico foi realizado com auxílio de espelho bucal plano (Golgran ${ }^{\circledR}$, São Paulo, Brazil), sonda exploradora no 5 (Golgran ${ }^{\circledR}$, São Paulo, Brazil) e sonda CPI (Golgran ${ }^{\circledR}$, São Paulo, Brazil) individuais devidamente esterilizados. O exame clínico de diagnóstico da cárie dentária foi realizado através do índice ceo- $\mathrm{d}^{17}$, que inclui o somatório do número de dentes decíduos cariados, com extração indicada e obturados.

\section{Análise Estatística}

Os dados foram analisados através do programa Statistical Package for the Social Science (SPSS ${ }^{\circledR}$ for Windows, versão 20.0, SPSS Inc., Chi- 
cago, IL, USA), sendo realizada a análise descritiva dos dados através de frequências, média $\mathrm{e}$ desvio-padrão. Foi aplicado o teste de Kolmogorov-Smirnov para testar a hipótese de distribuição de normalidade dos dados, a fim de definir o teste estatístico posterior.

$\mathrm{Na}$ análise multivariada, foram incluídas as variáveis que apresentaram valor de $\mathrm{p} \leq 0,20$ na análise bivariada, utilizando o modelo de Regressão de Poisson com variância robusta para determinar a relação entre qualidade de vida e variáveis selecionadas. Os resultados foram expressos por Razão de Taxas $(\mathrm{RR}=$ rate ratio $)$ e intervalo de $95 \%$ de confiança (IC95\%) e permaneceram no modelo as associações que alcançaram valor de $\mathrm{p}<0,05$. Em todas as análises foi considerado o nível de significância $\alpha=5 \%$.

\section{Resultados}

A amostra final foi constituída por 566 crianças $(94,0 \%)$ das 602 pré-estabelecidas. No dia do exame, 17 (2,8\%) crianças estavam ausentes e 15 $(2,5 \%)$ apresentaram idade superior a 05 anos. Quatro (04) crianças diagnosticadas com transtorno do espectro autista $(0,7 \%)$ foram excluídas de acordo com os critérios de elegibilidade. Os dados socioeconômicos e hábitos relativos à saúde bucal dos pesquisados estão descritos na Tabela 1.

Das crianças participantes da amostra, 50,2\% apresentaram experiência de cárie, sendo que $14,4 \%$ exibiram apenas os dentes anteriores afetados, $45,1 \%$ apenas os dentes posteriores e $40,5 \%$ dentes anteriores e posteriores. Referente à distribuição da necessidade de tratamento apenas 3,5\% não necessitavam de tratamento (Tabela 1$)$.

$\mathrm{Na}$ percepção das crianças, foi observado que indivíduos com renda familiar inferior a 2 salários mínimos apresentaram pior qualidade de vida de acordo com os domínios capacidade física e saúde bucal. Escolaridade dos pais menor que 8 anos de estudo formal foi associada a pior qualidade de vida no domínio saúde bucal. Além disso, observou-se que lesões cariosas localizadas em dentes posteriores afetaram a qualidade de vida no domínio capacidade física (Tabela 2).

A análise multivariada relativa à associação entre perfil socioeconômico, hábitos, experiência de cárie e localização das lesões cariosas sobre os domínios de qualidade de vida encontram-se descritas na Tabela 3. O sexo feminino foi associado à pior qualidade de vida nos domínios ca-
Tabela 1. Perfil socioeconômico, hábitos, experiência de cárie e localização em pré-escolares de Teresina (PI).

\begin{tabular}{|c|c|c|}
\hline Variáveis & $\mathbf{N}$ & $\%$ \\
\hline \multicolumn{3}{|l|}{ Sexo } \\
\hline Masculino & 301 & 53,2 \\
\hline Feminino & 265 & 46,8 \\
\hline \multicolumn{3}{|c|}{ Renda familiar (salários mínimos) } \\
\hline$<2 \mathrm{SM}$ & 382 & 67,5 \\
\hline
\end{tabular}

$184 \quad 32,5$

Escolaridade da mãe (anos de estudo formal)

$\leq 8$ anos

$>8$ anos

$150 \quad 26,5$

$416 \quad 73,5$

Escolaridade do pai (anos de estudo formal)

$\begin{array}{lcc}\leq 8 \text { anos } & 203 & 35,9 \\ >8 \text { anos } & 363 & 64,1 \\ \text { Não } & 9 & 1,6 \\ \text { Convive com pai e mãe na mesma } & & \\ \text { casa } & & \\ \text { Sim } & 398 & 70,3 \\ \text { Não } & 168 & 29,7 \\ \text { Tipo de escola } & & \\ \quad \text { Municipal } & 380 & 67,1 \\ \text { Particular } & 186 & 32,9\end{array}$

Quantas vezes a criança higieniza os dentes por dia?

\begin{tabular}{lcc}
1 & 58 & 10,2 \\
2 & 312 & 55,1 \\
$\geq 3$ & 196 & 34,6 \\
ngestão de guloseimas & & \\
$\quad$ Até 2 vezes por semana & 299 & 52,8 \\
3 ou mais vezes por semana & 267 & 47,2 \\
História de trauma dentário & & \\
nterior & & \\
Sim & 95 & 16,8 \\
Não & 471 & 83,2 \\
Experiência de cárie & & \\
Sim & 284 & 50,2 \\
Não & 282 & 49,8 \\
Localização (N=284) & & \\
Dentes anteriores & 41 & 14,4 \\
Dentes posteriores & 128 & 45,1 \\
Dentes anteriores e posteriores & 115 & 40,5 \\
Necessita de tratamento (N=284) & & \\
Sim & 274 & 96,5 \\
Não & 10 & 3,5 \\
\hline
\end{tabular}

pacidade física $(\mathrm{RR}=0,983 ; \mathrm{IC} 95 \%=0,97-0,99)$ e aspecto emocional $(\mathrm{RR}=0,984$; IC95\% $=0,98$ $0,99)$. Renda familiar menor que 2 salários míni- 
Tabela 2. Análise bivariada dos domínios de qualidade de vida das crianças segundo o perfil socioeconômico, hábitos, experiência de cárie e localização em pré-escolares de Teresina (PI).

\begin{tabular}{|c|c|c|c|c|c|c|c|c|c|c|}
\hline \multirow[t]{2}{*}{ Variáveis ${ }^{*}$} & \multicolumn{2}{|c|}{$\begin{array}{l}\text { Capacidade } \\
\text { física }\end{array}$} & \multicolumn{2}{|c|}{$\begin{array}{l}\text { Aspecto } \\
\text { emocional }\end{array}$} & \multicolumn{2}{|c|}{$\begin{array}{l}\text { Aspecto } \\
\text { social }\end{array}$} & \multicolumn{2}{|c|}{$\begin{array}{l}\text { Atividade } \\
\text { escolar }\end{array}$} & \multicolumn{2}{|c|}{$\begin{array}{l}\text { Saúde bucal } \\
\text { da criança }\end{array}$} \\
\hline & $\mu$ & DP & $\mu$ & DP & $\mu$ & DP & $\mu$ & DP & $\mu$ & DP \\
\hline \multicolumn{11}{|l|}{ Sexo } \\
\hline Masculino & 70,93 & 17,91 & 67,18 & 24,19 & 70,13 & 21,13 & 70,5 & 21,65 & 74,15 & 23,32 \\
\hline Feminino & 66,01 & 17,82 & 59,66 & 25,84 & 72,19 & 20,85 & 71,74 & 20,93 & 72,49 & 26,01 \\
\hline P-valor & \multicolumn{2}{|c|}{0,003} & \multicolumn{2}{|c|}{$<0,001$} & \multicolumn{2}{|c|}{$<0,001$} & \multicolumn{2}{|c|}{$<0,001$} & \multicolumn{2}{|c|}{0,495} \\
\hline \multicolumn{11}{|l|}{$\begin{array}{l}\text { Renda familiar (salários } \\
\text { mínimos) }\end{array}$} \\
\hline$<2 \mathrm{SM}$ & 66,66 & 18,23 & 61,26 & 25,76 & 70,34 & 21,14 & 69,84 & 22,67 & 70,47 & 25,62 \\
\hline$\geq 2 S M$ & 72,72 & 16,89 & 68,64 & 23,39 & 72,66 & 20,7 & 73,64 & 17,93 & 79,4 & 21,17 \\
\hline P-valor & \multicolumn{2}{|c|}{0,033} & \multicolumn{2}{|c|}{0,124} & \multicolumn{2}{|c|}{0,292} & \multicolumn{2}{|c|}{0,688} & \multicolumn{2}{|c|}{0,003} \\
\hline \multicolumn{11}{|c|}{$\begin{array}{l}\text { Escolaridade da mãe (anos de } \\
\text { estudo formal) }\end{array}$} \\
\hline$\leq 8$ anos & 63,88 & 17,72 & 58,87 & 26,46 & 66,93 & 22,16 & 65,67 & 21,47 & 64,47 & 26,13 \\
\hline$>8$ anos & 70,34 & 17,84 & 65,38 & 24,58 & 72,6 & 20,39 & 73,03 & 20,93 & 76,59 & 23,24 \\
\hline P-valor & \multicolumn{2}{|c|}{0,102} & \multicolumn{2}{|c|}{0,101} & \multicolumn{2}{|c|}{0,617} & \multicolumn{2}{|c|}{0,249} & \multicolumn{2}{|c|}{$<0,001$} \\
\hline \multicolumn{11}{|c|}{$\begin{array}{l}\text { Escolaridade do pai (anos de } \\
\text { estudo formal) }\end{array}$} \\
\hline$\leq 8$ anos & 65,21 & 18,42 & 61,72 & 25,48 & 68,57 & 22,81 & 68,92 & 23,14 & 67,44 & 25,65 \\
\hline$>8$ anos & 70,54 & 17,53 & 64,74 & 25,07 & 72,51 & 19,82 & 72,29 & 20,14 & 76,69 & 23,39 \\
\hline P-valor & \multicolumn{2}{|c|}{0,028} & \multicolumn{2}{|c|}{0,379} & \multicolumn{2}{|c|}{0,507} & \multicolumn{2}{|c|}{0,769} & \multicolumn{2}{|c|}{0,001} \\
\hline \multicolumn{11}{|l|}{ Experiência de cárie } \\
\hline Sim & 67,45 & 18,97 & 63,49 & 25,9 & 71,62 & 22,13 & 71,16 & 21,93 & 68,77 & 26,54 \\
\hline Não & 69,81 & 16,96 & 63,83 & 24,59 & 70,57 & 19,83 & 70,99 & 20,69 & 78,01 & 21,57 \\
\hline P-valor & & 19 & 0,8 & 71 & & 551 & & 25 & $<0$ & 001 \\
\hline Localização de cárie & & & & & & & & & & \\
\hline Anterior & 75,89 & 20,28 & 64,76 & 29,94 & 74,76 & 20,4 & 78,57 & 18,78 & 74,29 & 29,25 \\
\hline Posterior & 66,78 & 18,74 & 63,38 & 25,61 & 71,37 & 22,28 & 70,57 & 22,09 & 68,33 & 26,32 \\
\hline P-valor & & 29 & 0,8 & & & 657 & & 11 & 0,3 & 40 \\
\hline
\end{tabular}

*Teste de Poisson. $\mu=$ média. $\mathrm{DP}=$ desvio padrão.

mos, menor escolaridade dos pais e experiência de cárie dentária foram associados à pior qualidade de vida no aspecto saúde bucal da criança $(\mathrm{p}<0,05)$.

Na percepção dos pais, foi observada associação entre experiência de cárie e a escala de saúde bucal da criança no questionário $P e d s Q L^{T M}$ $(\mathrm{p}<0,001)$ (Tabela 4).

De acordo com a percepção dos pais, crianças com presença de experiência de cárie dentária apresentaram 5\% menor probabilidade de melhor qualidade de vida na escala de saúde bucal das crianças $(\mathrm{RR}=0,955 ; \mathrm{IC} 95 \%=0,94-0,97)$ (Tabela 5).

De acordo com a percepção dos pais, foi observada associação entre experiência de cárie e pior qualidade de vida no domínio de saúde bucal das crianças (Tabela 5).

\section{Discussão}

O conhecimento de dados sobre os efeitos de doenças bucais na qualidade de vida relacionada à saúde bucal de pré-escolares é de grande importância, pois permite a compreensão sobre os efeitos das condições que afetam a saúde bucal na vida dos mesmos e de suas famílias. Além disso, contribui para um entendimento mais completo das desigualdades sociais e seus efeitos para a identificação de grupos com maiores necessidades e para implantação de programas de saúde pública que priorizem a prevenção da cárie dentária e outros cuidados de saúde bucal para as crianças $^{18-21}$. A percepção de pais e responsáveis vem sendo utilizada para avaliar a qualidade de vida de seus filhos. Pais e responsáveis são considerados confiáveis quando se deseja avaliar QVR- 


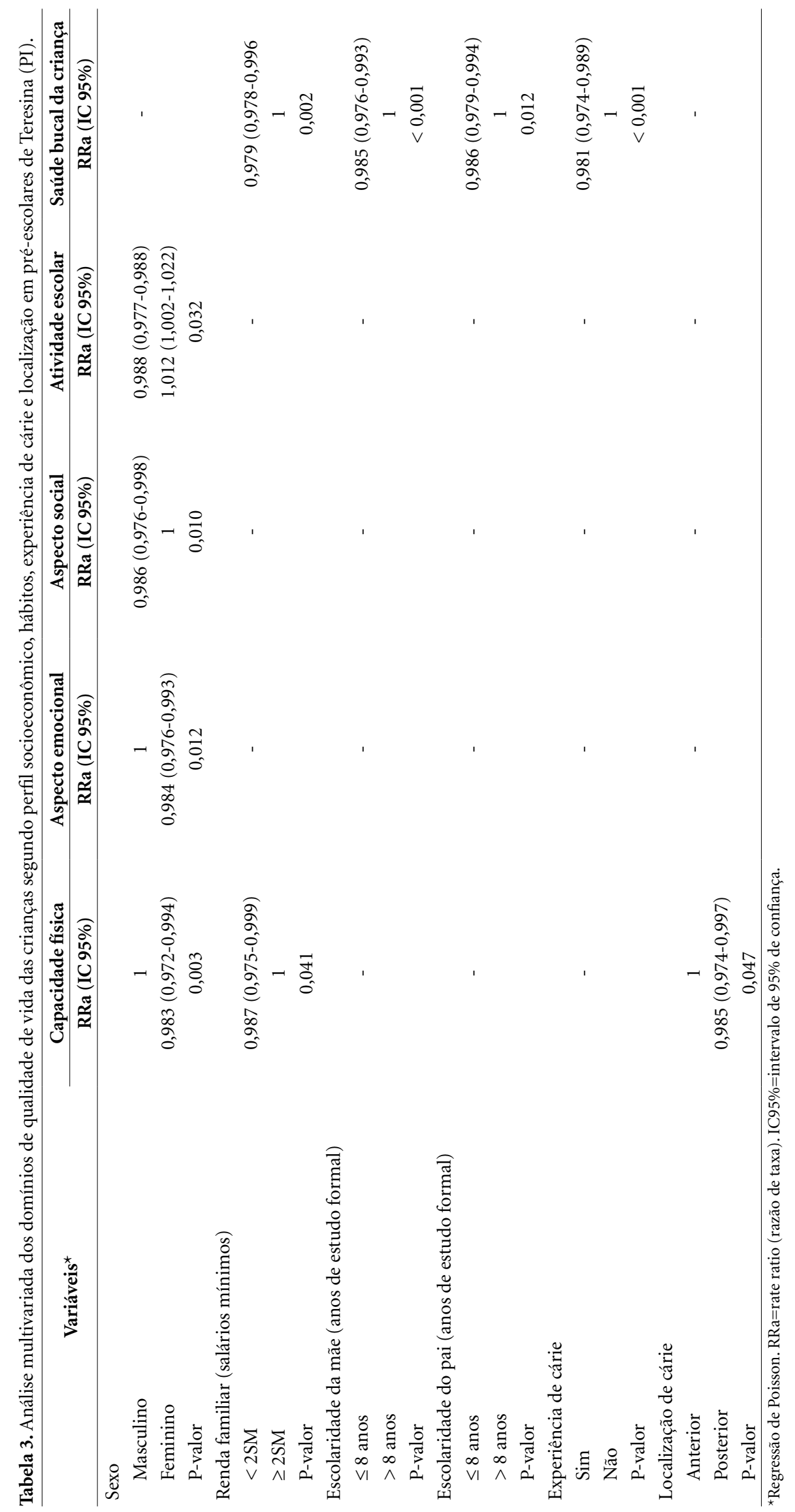


Tabela 4. Análise bivariada dos domínios de qualidade de vida das crianças conforme percepção dos pais segundo experiência de cárie e localização em pré-escolares de Teresina (PI).

\begin{tabular}{|c|c|c|c|c|c|c|c|c|c|c|}
\hline \multirow[t]{2}{*}{ Variáveis } & \multicolumn{2}{|c|}{$\begin{array}{c}\text { Capacidade } \\
\text { física }\end{array}$} & \multicolumn{2}{|c|}{$\begin{array}{c}\text { Aspecto } \\
\text { emocional }\end{array}$} & \multicolumn{2}{|c|}{ Aspecto social } & \multicolumn{2}{|c|}{$\begin{array}{c}\text { Atividade } \\
\text { escolar }\end{array}$} & \multicolumn{2}{|c|}{$\begin{array}{c}\text { Saúde bucal da } \\
\text { criança }\end{array}$} \\
\hline & $\mu$ & DP & $\mu$ & DP & $\mu$ & DP & $\mu$ & DP & $\mu$ & DP \\
\hline \multicolumn{11}{|c|}{ Experiência de cárie } \\
\hline Sim & 79,50 & 19,29 & 68,49 & 19,05 & 78,71 & 20,76 & 67,27 & 21,26 & 85,25 & 16,97 \\
\hline Não & 82,57 & 18,72 & 73,26 & 17,13 & 83,35 & 17,98 & 72,48 & 19,10 & 93,26 & 10,11 \\
\hline P-valor & \multicolumn{2}{|c|}{0,382} & \multicolumn{2}{|c|}{0,265} & \multicolumn{2}{|c|}{0,645} & \multicolumn{2}{|c|}{0,412} & \multicolumn{2}{|c|}{$<0,001$} \\
\hline \multicolumn{11}{|c|}{ Localização de cárie } \\
\hline Anterior & 76,04 & 21,77 & 66,90 & 15,77 & 74,05 & 23,59 & 66,19 & 17,46 & 87,14 & 16,17 \\
\hline Posterior & 79,78 & 19,10 & 68,61 & 19,31 & 79,09 & 20,53 & 67,36 & 21,56 & 85,10 & 17,06 \\
\hline P-valor & \multicolumn{2}{|c|}{0,498} & \multicolumn{2}{|c|}{0,498} & \multicolumn{2}{|c|}{0,358} & \multicolumn{2}{|c|}{0,706} & \multicolumn{2}{|c|}{0,550} \\
\hline
\end{tabular}

${ }^{\star}$ Teste de Poisson. $\mu=$ média. $\mathrm{DP}=$ desvio padrão.

Tabela 5. Análise multivariada dos domínios de qualidade de vida das crianças conforme percepção dos pais segundo experiência de cárie e localização em pré-escolares de Teresina (PI).

\begin{tabular}{|c|c|c|c|c|c|}
\hline \multirow[t]{2}{*}{ Variáveis } & $\begin{array}{c}\text { Capacidade } \\
\text { física } \\
\end{array}$ & $\begin{array}{c}\text { Aspecto } \\
\text { emocional }\end{array}$ & $\begin{array}{c}\text { Aspecto } \\
\text { social }\end{array}$ & $\begin{array}{c}\text { Atividade } \\
\text { escolar }\end{array}$ & $\begin{array}{c}\text { Saúde bucal da } \\
\text { criança }\end{array}$ \\
\hline & RRa (IC 95\%) & $\operatorname{RRa}(\mathrm{IC} 95 \%)$ & $\operatorname{RRa}(\mathrm{IC}$ 95\%) & $\operatorname{RRa}(\mathrm{IC} 95 \%)$ & RRa (IC 95\%) \\
\hline \multicolumn{6}{|c|}{ Experiência de cárie } \\
\hline Sim & & & & & $0,955(0,941-0,969)$ \\
\hline Não & - & - & - & - & 1 \\
\hline P-valor & & & & & $<0,001$ \\
\hline \multicolumn{6}{|c|}{ Localização de cárie } \\
\hline \multicolumn{6}{|l|}{ Anterior } \\
\hline Posterior & - & - & - & - & - \\
\hline P-valor & & & & & \\
\hline
\end{tabular}

SB de crianças de 5-6 anos de idade ${ }^{4,22}$. Resultado semelhante foi encontrado em nosso estudo, em que os pais de crianças com experiência de cárie têm a percepção que seus filhos têm pior qualidade de vida no escore saúde bucal da criança. Ao investigarem o impacto da cárie dentária na qualidade de vida de pré-escolares e de seus pais/ cuidadores, com ênfase sobre o tipo de dente e o estágio de progressão da lesão ${ }^{23}$, Ramos-Jorge et al. ${ }^{23}$ verificaram que a maioria das lesões de cárie apresentavam-se em estágio avançado e estavam localizadas tanto em dentes anteriores como posteriores. Naidu et al. ${ }^{24}$ também observaram em seu estudo que os maiores impactos negativos foram encontrados em crianças com maior gravidade de cárie. No presente estudo observou-se que presença de cárie em dentes posteriores foi associada à pior qualidade de vida no domínio capacidade física, isso pode ser devido à sensibilidade e à dor presente em dentes posteriores quando cariados, prejudicando a mastigação, levando à dificuldade de ingerir bebidas quentes ou frias e até mesmo problemas para dormir ${ }^{22}$. Além disso, outros autores afirmam que o impacto na qualidade de vida em crianças com experiência de cárie pode basear-se em fatores psicológicos, tendo em vista que crianças com cáries severas recordam suas experiências ruins com relação à dor e ansiedade no tratamento ${ }^{2,17,25}$.

A grande incidência e prevalência de cárie dentária em pré-escolares justificaram o desenvolvimento desta pesquisa, pois ainda se observa carência de informações sobre a temática, no que diz respeito a dados estatísticos do município de Teresina, bem como sobre o impacto da cárie dentária na qualidade de vida de pré-escolares. 
No entanto, estudos longitudinais são necessários para determinar a relação causa-efeito entre cárie e qualidade de vida.

Em revisão sistemática da literatura realizada por Barbosa e Gavião ${ }^{26}$, os autores concluíram que existe relação entre a saúde bucal e QVRSB em crianças. Condições como cárie dental, fluorose e maloclusão impactam negativamente na qualidade de vida das crianças. Entretanto, os autores sugeriram que estudos para avaliar outras condições bucais devem ser realizados para maximizar a validade dos instrumentos. Resultados semelhantes foram encontrados em outros estudos s $^{2,26-29}$.

Em nosso estudo, observou-se alta prevalência de cárie dentária na amostra, foi possível identificar que 50,2\% apresentaram experiência de cárie, sendo que $14,6 \%$ exibiram apenas os dentes anteriores afetados, $45,1 \%$ apenas os dentes posteriores e $40,6 \%$ dentes anteriores e posteriores. Esses dados corroboram os dados encontrados no SB Brasil ${ }^{30}$, em que a média de ceo-d na idade de 5 anos é de 2,55 e 56,7\% apresentaram experiência de cárie na cidade de Teresina-PI. Ao avaliarmos a associação entre experiência de cárie e os domínios da qualidade de vida das crianças em suas percepções, apenas o domínio de saúde bucal das crianças apresentou diferença significativa ( $p>0,001)$ quanto à existência de cárie, em se tratando da variável sexo. Foi observada associação entre os domínios capacidade física, aspecto emocional, aspecto social e atividade escolar e sexo das crianças, demonstrando que as características de gênero podem afetar as condições físicas, sociais e emocionais das crianças.

Estudo realizado por Martins Júnior et al. ${ }^{31}$ concluiu que a variável renda familiar não influenciou na presença de cárie nas crianças daquele estudo, diferindo do presente estudo, em que se observou que nas famílias com renda inferior a 02 salários mínimos, as crianças apresentaram pior QVRSB.

É importante que os pais ou responsáveis tenham conhecimento sobre as condições que afetam a saúde bucal de seus filhos, porém isso nem sempre é possível, pois pode estar relacionado ao nível de escolaridade dos mesmos. No presente estudo, verificou-se que filhos de pais que possuíam maior nível de escolaridade, apresentaram melhor saúde bucal e qualidade de vida. Esse resultado corrobora o encontrado por Gomes et al. ${ }^{4}$, e Martins ${ }^{32}$ e contraria Firmino et al. ${ }^{33}$. É importante a promoção de saúde bucal durante a infância, pois fatores de risco como dieta rica em açucares, má higiene bucal e condições socioeconômicas desfavoráveis podem contribuir para aumentar as chances de impacto negativo na qualidade de vida ${ }^{34-38}$.

Observou-se associação entre experiência de cárie e pior qualidade de vida no domínio de saúde bucal tanto na percepção das crianças quanto dos pais. Os pais que tem conhecimento sobre a cárie dentária apresentam filhos com menores índices de prevalência, diferindo do que foi encontrado no estudo de Firmino et al. ${ }^{33}$, em que não foram encontradas diferenças significativas nas condições de saúde bucal e qualidade de vida das crianças em que os pais tinham um histórico de conhecimento prévio sobre cárie e que visitavam o dentista regularmente. Tendo em vista que a maioria das consultas odontológicas tem enfoque curativo ${ }^{9}$ é fundamental o estabelecimento de ações educativas voltadas aos pais para prevenção das doenças bucais desde a infância.

A versão brasileira validada do questionário PedsQL ${ }^{\mathrm{TM}}$ foi utilizada pois permite fazer comparações entre as percepções dos responsáveis e das crianças, além de possibilitar avaliação longitudinal da qualidade de vida relacionada à saúde geral e bucal ${ }^{12}$. Além disso, as pontuações desse instrumento foram analisadas como variáveis quantitativas por meio da regressão de Poisson com variância robusta bivariada e multivariada, como realizado em estudos anteriores ${ }^{39,40}$.

Como uma avaliação subjetiva, o PedsQL permite que os pais/responsáveis tenham maior consciência do impacto que os problemas orais têm sobre seus filhos, tendo em vista que a saúde bucal dos pré-escolares é muitas vezes negligenciada ou colocada em uma posição secundária. Crianças com cárie não tratada experimentam dor, além de dificuldades em mastigar, dormir e socializar, com possíveis efeitos na autoestima, crescimento, ganho de peso e qualidade de vida $^{8,20,38,41}$. Este estudo é relevante, pois os resultados podem aumentar a consciência dos pais/ responsáveis e estimular medidas mais eficazes de promoção e prevenção, através de politicas públicas de saúde bucal, visto que utiliza a percepção dos pais e responsáveis, evidenciando dados mais concretos sobre o impacto de problemas orais sobre a rotina de pré-escolares.

\section{Conclusão}

Experiência de cárie dentária impactou negativamente a qualidade de vida relacionada à saúde bucal na escala de saúde bucal de crianças pré-escolares no questionário PedsQL, de acordo com a percepção das crianças e dos seus pais. 


\section{Colaboradores}

AV Nóbrega trabalhou na concepção, pesquisa, metodologia e na redação final; LFAD Moura trabalhou na concepção e na redação final; NS Andrade trabalhou na pesquisa e na metodologia; CCB Lima trabalhou na pesquisa e na metodologia; DG Dourado trabalhou na redação final; MDM Lima trabalhou na concepção, pesquisa, metodologia e na redação final.

\section{Referências}

1. Wong HM, Mcgrath CPJ, King NM, Lo ECM. Oral Health-Related Quality of Life in Hong Kong Preschool Children. Caries Research 2011;45(4):370-376.

2. Kramer PF, Feldens CA, Ferreira SH, Bervian J, Rodrigues $\mathrm{PH}$, Peres MA. Exploring the impact of oral diseases and disorders on quality of life of preschool children. Community Dent Oral Epidemiol 2013;41(4):327-335.

3. Scarpelli AC, Paiva SM, Viegas CM, Carvalho AC, Ferreira FM, Pordeus IA. Oral health-related quality of life among Brazilian preschool children. Community Dent Oral Epidemiol 2013;41(4):336-344.

4. Gomes MC, Pinto-Sarmento TCDA, Costa EMMDB, Martins CC, Granville-Garcia AF, Paiva SM. Impact of oral health conditions on the quality of life of preschool children and their families: a cross-sectional study. Health Qual Life Outcomes 2014;18(12):55-67.

5. Pereira SM, Tagliaferro EP, Ambrosano GM, Cortelazzi KL, Meneghim MC, Pereira AC. Dental caries in 12-year-old schoolchildren and its relationship with socioeconomic and behavioural variables. Oral Health Prev Dent 2007;5(4):299-306.

6. Traebert J, Guimarães LA, Durante EZ, Serratine AC. Low maternal schooling and severity of dental caries in Brazilian preschool children. Oral Health Prev Dent 2009;7(1):39-45.

7. Cerveira JA. Influência da qualidade de vida na ocorrência da doença carie em pré-escolares [mestrado]. São Paulo: Escola de enfermagem de Ribeirão Preto - Universidade de São Paulo; 2003.

8. Feitosa S, Colares V. As repercussões da cárie precoce na infância na qualidade de vida de pré-escolares. JBP Rev Ibero-Am Odontopediatr Odontol Bebê 2003;6(34):542-548.

9. Slade GD, Strauss RP, Atchison KA, Kressin NR, Locker D, Resine ST. Conference summary: assessing oral health outcomes - measuring heath status and quality of life. Community Dent Health 1998;15(1):3-7.

10. Pahel, BT, Rozier RG, Slade GD. Parental perceptions of children's oral health: the Early Childhood Oral Health Impact Scale (ECOHIS). Health Qual Life Outcomes 2007;5(6):1-10.

11. Sischo L, Broder HL. Oral health-related quality of life: what, why, how and future. J Dent Res 2011;90(11):1264-1270.

12. Bendo CB, Paiva SM, Viegas CM, Vale MP, Varni JW. The PedsQL ${ }^{\text {тм }}$ Oral Health Scale: feasibility, reliability and validity of the Brazilian Portuguese version. Health Qual Life Outcomes 2012;10:42-53.

13. Talekar BS, Rozier RG, Slade GD, Ennett ST. Parental perceptions of their preschool-aged children's oral health. J Am Dent Assoc 2005;136(3):364-372.

14. Brasil. Ministério da Saúde. Conselho Nacional de Saúde. Resolução no 466, de 12 de dezembro de 2012. Brasília: DOU no 12, Seção 1, 2012.

15. Varni JW, Seid M, Kurtin PS. PedsQL 4.0: reliability and validity of the Pediatric Quality of Life Inventory version 4.0 generic core scales in healthy and patient populations. Med Care 2001;39(8):800-812.

16. Steele MM, Steele RG, Varni JW. Reliability and validity of the PedsQL ${ }^{\mathrm{TM}}$ Oral Health Scale: measuring the relationship between child oral health and health-related quality of life. Child Health Care 2009;38:228-244. 
17. WHO. The World Oral Health Report. Continuous im provement of oral health in the $21^{\text {st }}$ century - The approach of the WHO Global Oral Health Programme. Geneva: World Health Organization; 2003.

18. Fernandes IB, Pereira TS, Souza DS, Ramos-Jorge J, Marques LS, Ramos-Jorge ML. Severity of Dental Caries and Quality of Life for Toddlers and Their Families. Pediatr Dent 2017;39(2):118-123.

19. Kragt L, Van Der Tas JT, Moll HA, Elfrink ME, Jaddoe VW, Wolvius EB, Ongkosuwito EM. Early Caries Predicts Low Oral Health-Related Quality of Life at a Later Age. Caries Res 2016;50(5):471-479.

20. Lee GH, Mcgrath C, Yiu CK, King NM. Translation and validation of a Chinese language version of the Early Childhood Oral Health Impact Scale (ECOHIS). Int J Paediatr Dent 2009;19(6):399-405.

21. Ferreira SH, Béria JU, Kramer PF, Feldens EG, Feldens CA. Dental caries in 0- to 5-year-old Brazilian children: prevalence, severity, and associated factors. Int $J$ Paediatr Dent 2007;17(4):289-296.

22. Abanto J, Tsakos G, Paiva SM, Raggio DP, Celiberti P, Bonecker M. Agreement between children aged 5-6 years and their mothers in rating child oral health-related quality of life. Int J Paediatr Dent 2014; 24(5):373-379.

23. Ramos-Jorge J, Alencar BM, Pordeus IA, Soares MEC Marques LS, Ramos-Jorge ML, Paiva SM. Impact of dental caries on quality of life among preschool children: emphasis on the type of tooth and stages of progression. Int J Paediatr Dent 2014;24(5):373-379.

24. Naidu R, Nunn J, Donnelly-Swift E. Oral health-related quality of life and early childhood caries among preschool children in Trinidad. BMC Oral Health 2016;16(1):128

25. Peres MA, De Oliveira Latorre MDOR, Sheiham A, Peres KG, Barros FC, Hernandez PG, Maas AM, Romano AR, Victora CG. Social and biological early life influences on severity of dental caries in children aged 6 years. Community Dent Oral Epidemiol 2005;33:5363.

26. Barbosa TS, Gaviao MB. Oral health-related quality of life in children: part II. Effects of clinical oral health status. A systematic review. Int J Dent Hyg 2008;6(2):100-107.

27. Chaffee BW, Kramer PF, Vítolo MR, Feldens CA. Oral health-related quality-of-life scores differ by socioeconomic status and caries experience. Community Dent Oral Epidemiol 2017;45(3):216-224.

28. Guerra MJC, Greco RM, Leite ICG, Ferreira EF, Paula MVQ. Impacto das condições de saúde bucal na qualidade de vida de trabalhadores. Ciên Saude Colet 2014;19(12):4777-4786.

29. Lopes MWF, Gusmão ES, Alves RV, Cimões R. Impacto das doenças periodontais na qualidade de vida. RGO - Rev Gaúcha Odontol 2011;59(supl. 0):39-44.

30. Brasil. Ministério da Saúde. SB Brasil 2010: Pesquisa Nacional de Saúde Bucal: resultados principais. Brasília: Ministério da Saúde; 2012.

31. Martins-Júnior PA, Vieira-Andrade RG, Corrêa-Faria P, Oliveira-Ferreira F, Marques LS, Ramos-Jorge ML. Impact of Early Childhood Caries on the Oral Health-Related Quality of Life of Preschool Children and Their Parents. Caries Research 2013;47(3):211-218.
32. Martins MT. Dental caries and social factors: impact on quality of life in Brazilian children. Braz Oral Res 2015;29(sup. 1):1-7.

33. Firmino RT, Gomes MC, Clementino MA, Martins CC, Paiva SM, Granville-Garcia AF. Impact of oral health problems on the quality of life of preschool children: a case-control study. Int J Paediatr Dent 2016;26(4):242-249.

34. Casamassimo PS, Thikkurissy S, Edelstein BL, Maiorini E. Beyond the dmft: The Human and Economic Cost of Early Childhood Caries. J Am Dent Assoc 2009;140(6):650-657.

35. Freire MCM, Reis SCGB, Figueiredo N, Peres KG, Moreira RS, Antunes JLF. Determinantes individuais e contextuais da carie em crianças brasileiras de 12 anos em 2010. Rev Saude Publica 2013;47(supl. 3):40-49.

36. Narvai PC, Frazão P, Roncalli AG, Antunes JLF. Cárie dentária no Brasil: declínio, polarização, iniqüidade e exclusão social. Rev Panam Salud Publica 2006;19(6):385-393.

37. Martins CLC, Jetelina JC. Conhecimento dos pais sobre saúde bucal na infância e a relação com o motivo da consulta odontológica. J Oral Invest 2016;5(1):2733.

38. Oliveira LB, Sheiham A, B onecker M. Exploring the association of dental caries with social factors and nutritional status in Brazilian preschool children. Eur J Oral Sci 2008;116(1):37-43.

39. Abanto J, Paiva SM, Raggio DP, Celiberti P, Aldrigui JM, Bonecker M. The impact of dental caries and trauma in children on family quality of life. Community Dent Oral Epidemiol 2012;40:323-331.

40. Bendo CB, Paiva SM, Abreu MH, Figueiredo LD, Vale MP. Impact of traumatic dental injuries among adolescents on family's quality of life: a population-based study. Int J Paediatr Dent 2014;24:387-396.

41. Abanto J, Carvalho TS, Mendes FM, Wanderley MT, Bönecker M, Raggio DP. Impact of oral diseases and disorders on oral health-related quality of life of preschool children. Community Dent Oral Epidemiol 2011;39(2):105-114.

Artigo apresentado em 04/09/2017

Aprovado em 09/04/2018

Versão final apresentada em 11/04/2018 
